\title{
THE ZEROS OF FABER POLYNOMIALS GENERATED BY AN $m$-STAR
}

\author{
ARNO B. J. KUIJLAARS
}

\begin{abstract}
It is shown that the zeros of the Faber polynomials generated by a regular $m$-star are located on the $m$-star. This proves a recent conjecture of J. Bartolomeo and M. He. The proof uses the connection between zeros of Faber polynomials and Chebyshev quadrature formulas.
\end{abstract}

\section{INTRODUCTION}

Let $\mathbf{C}$ denote the complex plane, $\overline{\mathbf{C}}=\mathbf{C} \cup\{\infty\}$, and let $E$ be a compact set in the complex plane (not a single point) such that $\overline{\mathbf{C}} \backslash E$ is simply connected. Let $\phi$ denote the conformal mapping from $\mathbf{C} \backslash E$ onto $|w|>\rho$ such that in a neighborhood of $\infty$,

$$
\phi(z)=z+a_{0}+\frac{a_{1}}{z}+\frac{a_{2}}{z^{2}}+\cdots .
$$

Here, $\rho=\rho_{E}$ is the logarithmic capacity of $E$. The polynomial part of $\phi(z)^{n}$ is called the Faber polynomial of degree $n$ generated by $E$.

Bartolomeo and He [1] studied the Faber polynomials generated by the regular $m$-star

$$
S_{m}:=\left\{z \in \mathbf{C} \mid z^{m} \in[0,4]\right\}, \quad m=2,3, \ldots .
$$

They obtained several properties of these Faber polynomials and they formulated a conjecture on their zeros. Here we will prove this conjecture.

Theorem 1. Let $m \geq 2, n \geq 1$, and let $F_{n}$ be the Faber polynomial of degree $n$ generated by $S_{m}$. Then all the zeros of $F_{n}$ are located on $S_{m}$.

The proof of Theorem 1 is based on the connection between zeros of Faber polynomials and quadrature formulas with equal weights (so-called Chebyshev quadrature formulas), see Lemma 3 below. This connection was used before to obtain results on Chebyshev quadrature from properties of Faber polynomials, see $[4,5,7]$. Here we use this connection in the opposite direction. We will obtain Theorem 1 from the following quadrature result.

Received by the editor June 20, 1994.

1991 Mathematics Subject Classification. Primary 30C15; Secondary 65D32.

Key words and phrases. Faber polynomials, zero distribution, Chebyshev quadrature.

Supported by the Netherlands Foundation for Mathematics SMC with financial aid from the Netherlands Organization for the Advancement of Scientific Research NWO.

(C)1996 American Mathematical Society 
Theorem 2. Let $p \in \mathbf{N}$ and $\alpha \in[0,1 /(p+1)]$. Then there exist nodes $t_{1}, \ldots, t_{p}$ in $[0,1]$ such that

$$
\int_{0}^{1} f(t) \frac{d t}{\pi \sqrt{t-t^{2}}}=\alpha f(0)+\frac{1-\alpha}{p} \sum_{j=1}^{p} f\left(t_{j}\right)
$$

for all polynomials $f$ of degree $\leq p$.

Note that for $\alpha=0$ the $p$-point Gauss formula with respect to $d t /\left(\pi \sqrt{t-t^{2}}\right)$ can be used in (1.1). This formula is exact for all polynomials up to degree $2 p-1$. A quadrature formula (1.1) for some $\alpha \geq 0$ in which all the nodes are distinct can be perturbed a little to give a quadrature formula for slightly larger $\alpha$. So our main concern in proving Theorem 2 will be to show that for $\alpha<1 /(p+1)$ the nodes do not coincide.

\section{Proof that Theorem 2 implies Theorem 1}

Let $E$ be a compact set whose complement is simply connected with respect to the extended complex plane. Let $\rho$ be the logarithmic capacity of $E$ and $\phi$ the conformal mapping from $\mathbf{C} \backslash E$ onto $|w|>\rho$ such that $\phi(\infty)=\infty$ and $\phi^{\prime}(\infty)=1$. We denote by $\mu_{E}$ the equilibrium measure on $E$. This is the unique probability measure on $E$ satisfying

$$
\int \log |z-\zeta| d \mu_{E}(\zeta)= \begin{cases}\log \rho_{E} & \text { quasi-everywhere on } E, \\ \log |\phi(z)| & \text { on } \mathbf{C} \backslash E .\end{cases}
$$

Lemma 3. Let $n \geq 1$, and let $\zeta_{1}, \ldots, \zeta_{n} \in \mathbf{C}$. Then $\zeta_{1}, \ldots, \zeta_{n}$ are the zeros of the Faber polynomial of degree $n$ generated by $E$ if and only if

$$
\int \zeta^{k} d \mu_{E}=\frac{1}{n} \sum_{j=1}^{n} \zeta_{j}^{k}, \quad k=1, \ldots, n .
$$

Proof. The function $\phi(z) / z$ is analytic in $\overline{\mathbf{C}} \backslash E$ (including $\infty$ ), and from (2.1) it is easy to see that

$$
\log \left(\frac{\phi(z)}{z}\right)=\int \log \left(1-\frac{\zeta}{z}\right) d \mu_{E}(\zeta)=-\sum_{k=1}^{\infty} \frac{1}{k} \int \zeta^{k} d \mu_{E} z^{-k}
$$

Here we take the branch of the logarithm that vanishes at $\infty$. Also, if $F_{n}(z)=$ $\prod_{j=1}^{n}\left(z-\zeta_{j}\right)$, then

$$
\log \left(\frac{F_{n}(z)}{z^{n}}\right)=\sum_{j=1}^{n} \log \left(1-\frac{\zeta_{j}}{z}\right)=-\sum_{k=1}^{\infty} \frac{1}{k} \sum_{j=1}^{n} \zeta_{j}^{k} z^{-k} .
$$

It follows that (2.2) holds if and only if

$$
\log \left(\frac{F_{n}(z)}{z^{n}}\right)=n \log \left(\frac{\phi(z)}{z}\right)+\mathcal{O}\left(z^{-n-1}\right), \quad z \rightarrow \infty,
$$

and this holds if and only if

$$
F_{n}(z)=\phi(z)^{n}+\mathcal{O}\left(z^{-1}\right), \quad z \rightarrow \infty
$$

that is, $F_{n}(z)$ is the Faber polynomial of degree $n$ generated by $E$. 
Let $F_{n}(z)$ be the Faber polynomial of degree $n$ generated by $S_{m}$. Write $n=$ $p m+r$ with $0 \leq r<m$. From the symmetry of $S_{m}$ it easily follows that

$$
F_{n}(z)=z^{r} G_{p}\left(z^{m} / 4\right)
$$

where $G_{p}$ is a polynomial of degree $p$. The zeros of $F_{n}$ are on $S_{m}$ if and only if the zeros of $G_{p}$ are in the interval $[0,1]$.

Lemma 4. The zeros $t_{1}, \ldots, t_{p}$ of $G_{p}$ satisfy

$$
\int_{0}^{1} t^{k} \frac{d t}{\pi \sqrt{t-t^{2}}}=\frac{m}{n} \sum_{j=1}^{p} t_{j}^{k}, \quad k=1, \ldots, p .
$$

Conversely, if $t_{1}, \ldots, t_{p}$ are such that $(2.3)$ holds, then $t_{1}, \ldots, t_{p}$ are the zeros of $G_{p}$.

Proof. Let $t_{1}, \ldots, t_{p}$ be the zeros of $G_{p}$. Then $\left(4 t_{j}\right)^{1 / m} \exp (2 \pi i l / m), j=1, \ldots, p$, $l=1, \ldots, m$, are the zeros of $F_{n}$, together with an $r$-fold zero at the origin. Thus, if $\zeta_{1}, \ldots, \zeta_{n}$ are the zeros of $F_{n}$, then for every $k$

$$
\frac{1}{n} \sum_{j=1}^{n} \zeta_{j}^{k m}=4^{k} \frac{m}{n} \sum_{j=1}^{p} t_{j}^{k} .
$$

Then by Lemma 3,

$$
\frac{m}{n} \sum_{j=1}^{p} t_{j}^{k}=4^{-k} \int \zeta^{k m} d \mu_{S_{m}}(\zeta), \quad k=1, \ldots, p .
$$

The moments of $\mu_{S_{m}}$ were computed in [1]:

$$
\int \zeta^{k m} d \mu_{S_{m}}(\zeta)=\frac{m}{\pi} \int_{0}^{4^{1 / m}} s^{k m} \frac{s^{(m-2) / 2}}{\sqrt{4-s^{m}}} d s=\frac{4^{k}}{\pi} \int_{0}^{1} t^{k} \frac{d t}{\sqrt{t-t^{2}}},
$$

where we have put $s^{m}=4 t$. Now (2.3) follows from (2.5) and (2.6).

For the converse, suppose $t_{1}, \ldots, t_{p}$ satisfy $(2.3)$. Let $\zeta_{1}, \ldots, \zeta_{n}$ be the zeros of $z^{r} \prod_{j=1}^{p}\left(z^{m} / 4-t_{j}\right)$. Then it is easy to see that (2.4) holds. From (2.3), (2.4) and (2.6) it follows that

$$
\frac{1}{n} \sum_{j=1}^{n} \zeta_{j}^{k m}=\int \zeta^{k m} d \mu_{S_{m}}, \quad k=1, \ldots, p,
$$

i.e., (2.2) holds if $k \leq n$ is a multiple of $m$. By the symmetry of $S_{m}$ and the points $\zeta_{1}, \ldots, \zeta_{n}$ both sides of (2.2) are zero if $k$ is not a multiple of $m$. Hence, (2.2) holds and it follows from Lemma 3 that $\zeta_{1}, \ldots, \zeta_{n}$ are the zeros of $F_{n}$. Then $t_{1}, \ldots, t_{p}$ are the zeros of $G_{p}$.

Corollary 5. Theorem 2 implies Theorem 1.

Proof. Let $m \geq 2, n \geq 1$ and write $n=p m+r$ with $0 \leq r<m$. Let $F_{n}(z)=$ $z^{r} G_{p}\left(z^{m} / 4\right)$ be the Faber polynomial of degree $n$ generated by $S_{m}$. Let $\alpha:=r / n<$ $1 /(p+1)$. By Theorem 2 there are nodes $t_{1}, \ldots, t_{p}$ in $[0,1]$ such that $(1.1)$ holds for polynomials of degree $\leq p$. Since $(1-\alpha) / p=m / n$, it follows that $(2.3)$ holds and therefore, by Lemma $4, t_{1}, \ldots, t_{p}$ are the zeros of $G_{p}$. Thus, the zeros of $G_{p}$ are in $[0,1]$, and therefore the zeros of $F_{n}$ are on $S_{m}$. 


\section{Proof of Theorem 2}

We first discuss the maximal mass function $\lambda_{p}(t)$ with respect to the measure $d t /\left(\pi \sqrt{t-t^{2}}\right)$ on $[0,1]$, cf. [3, §IV.3].

Let $p \in \mathbf{N}$ be fixed. Consider all quadrature formulas

$$
\int_{0}^{1} f(t) \frac{d t}{\pi \sqrt{t-t^{2}}} \approx \sum_{j=1}^{N} c_{j} f\left(t_{j}\right)
$$

that are exact for polynomials $f$ of degree $\leq p$ and are such that

$$
c_{j} \geq 0, \quad t_{j} \in[0,1], \quad j=1, \ldots, N .
$$

We call $c_{j}$ the mass at $t_{j}$.

Definition 6. For $t \in[0,1]$, the number $\lambda_{p}(t)$ is defined as the maximal mass at $t$ among all such quadrature formulas.

We need the following result.

Lemma 7. The following estimate holds

$$
\lambda_{p}(t) \leq \frac{2}{p+1}, \quad t \in[0,1] .
$$

In addition we have

$$
\lambda_{p}(0)=\lambda_{p}(1)=\frac{1}{p+1} .
$$

Proof. First, let $p=2 q-1$. There exist two principal quadrature formulas (cf. [3]), namely the familiar $q$-point Gauss formula

$$
\int_{0}^{1} f(t) \frac{d t}{\pi \sqrt{t-t^{2}}} \approx \frac{2}{p+1} \sum_{j=1}^{q} f\left(t_{j}^{*}\right)
$$

and the Lobatto formula (or Bouzitat formula of second kind), cf. [2, pp. 106-108],

$$
\int_{0}^{1} f(t) \frac{d t}{\pi \sqrt{t-t^{2}}} \approx \frac{1}{p+1} f(0)+\frac{1}{p+1} f(1)+\frac{2}{p+1} \sum_{j=1}^{q-1} f\left(s_{j}^{*}\right) .
$$

The nodes $t_{j}^{*}$ are the zeros of $T_{q}(2 t-1)$, where $T_{q}$ is the Chebyshev polynomial of the first kind of degree $q$ and the nodes $s_{j}^{*}$ are the zeros of $U_{q-1}(2 t-1)$, where $U_{q-1}$ is the Chebyshev polynomial of the second kind of degree $q-1$. We note the separation property

$$
0<t_{1}^{*}<s_{1}^{*}<t_{2}^{*}<\cdots<s_{q-1}^{*}<t_{q}^{*}<1
$$

The Gauss and Lobatto formulas are exact for polynomials of degree $\leq p$ and have maximal mass at all of their nodes, i.e.,

$$
\begin{gathered}
\lambda_{p}(0)=\lambda_{p}(-1)=\frac{1}{p+1}, \\
\lambda_{p}\left(t_{1}^{*}\right)=\cdots=\lambda_{p}\left(t_{q}^{*}\right)=\lambda_{p}\left(s_{1}^{*}\right)=\cdots=\lambda_{p}\left(s_{q-1}^{*}\right)=\frac{2}{p+1} .
\end{gathered}
$$

Next, by a result of Schoenberg and Szegö [6],

$$
\frac{1}{\lambda_{p}(t)}=\max \{P(t), Q(t)\}
$$


where $P$ and $Q$ are two polynomials of degree $p$. These polynomials have the common value $(p+1) / 2$ in the points $t_{1}^{*}, \ldots, t_{q}^{*}, s_{1}^{*}, \ldots, s_{q-1}^{*}$, and at the end points we have

$$
P(0)=p+1, \quad Q(0)=0, \quad P(1)=0, \quad Q(1)=p+1 .
$$

See [3] or [6] for the precise form of $P$ and $Q$.

It follows that $P(t)>(p+1) / 2$ on the intervals $\left(0, t_{1}^{*}\right)$ and $\left(s_{j}^{*}, t_{j+1}^{*}\right), j=$ $1, \ldots, q-1$, while $Q(t)>(p+1) / 2$ on the remaining intervals $\left(t_{j}^{*}, s_{j}^{*}\right), j=1, \ldots, q-$ 1 , and $\left(t_{q}^{*}, 1\right)$. Hence, for every $t \in[0,1]$,

$$
\frac{1}{\lambda_{p}(t)}=\max \{P(t), Q(t)\} \geq \frac{p+1}{2} .
$$

This proves the lemma in case $p$ is odd.

For $p=2 q$ one has to proceed in a similar way, but the details are slightly different. The two principal quadrature formulas are Radau formulas (or Bouzitat formulas of first kind), cf. [2, pp. 101-103],

$$
\int_{0}^{1} f(t) \frac{d t}{\pi \sqrt{t-t^{2}}} \approx \frac{1}{p+1} f(0)+\frac{2}{p+1} \sum_{j=1}^{q} f\left(t_{j}^{*}\right)
$$

and

$$
\int_{0}^{1} f(t) \frac{d t}{\pi \sqrt{t-t^{2}}} \approx \frac{1}{p+1} f(1)+\frac{2}{p+1} \sum_{j=1}^{q} f\left(s_{j}^{*}\right),
$$

with separation property

$$
0<s_{1}^{*}<t_{1}^{*}<\cdots<s_{q}^{*}<t_{q}^{*}<1 .
$$

The nodes $t_{j}^{*}$ and $s_{j}^{*}$ are the zeros of $P_{q}^{(-1 / 2,1 / 2)}(2 t-1)$ and $P_{q}^{(1 / 2,-1 / 2)}(2 t-1)$, respectively, where $P_{q}^{(\alpha, \beta)}$ denotes a Jacobi polynomial. Again, these formulas are exact for polynomials of degree $\leq p$ and have maximal mass at all of their nodes. Hence,

$$
\begin{gathered}
\lambda_{p}(0)=\lambda_{p}(-1)=\frac{1}{p+1} \\
\lambda_{p}\left(t_{1}^{*}\right)=\cdots=\lambda_{p}\left(t_{q}^{*}\right)=\lambda_{p}\left(s_{1}^{*}\right)=\cdots=\lambda_{p}\left(s_{q}^{*}\right)=\frac{2}{p+1} .
\end{gathered}
$$

The rest of the proof is the same as in the case of odd $p$.

Proof of Theorem 2. Let $p \in \mathbf{N}$. We observe first that for $\alpha=0$ the $p$-point Gauss formula gives a quadrature formula (1.1) with nodes in $(0,1)$.

Next, we note that the statement that (1.1) holds for every polynomial of degree $\leq p$ is equivalent to

$$
\sum_{j=1}^{p} t_{j}^{k}=\frac{p}{1-\alpha} \int_{0}^{1} t^{k} \frac{d t}{\pi \sqrt{t-t^{2}}}, \quad k=1, \ldots, p .
$$

It is easy to see that the mapping $\left(t_{1}, \ldots, t_{p}\right) \mapsto\left(s_{1}, \ldots, s_{p}\right)$, where $s_{k}=\sum_{j=1}^{p} t_{j}^{k}$, is locally surjective if the points $t_{1}, \ldots, t_{p}$ are distinct. Since for $\alpha=0$ we have distinct nodes in $(0,1)$, it follows that for $\alpha>0$ sufficiently small, there exist nodes $t_{1}, \ldots, t_{p}$ in $(0,1)$ satisfying $(3.3)$ and hence (1.1). In fact, we can continue this process until for some $\alpha$, we find a solution of (1.1) in which either two nodes coincide or one of the nodes coincides with one of the end points. 
Thus, we shall be finished if we can show that if the points $t_{1}, \ldots, t_{p}$ satisfy $(1.1)$ with $\alpha<1 /(p+1)$ and

$$
0 \leq t_{1} \leq t_{2} \leq \cdots \leq t_{p} \leq 1
$$

then we must have strict inequalities everywhere in (3.4).

This follows, however, quite easily from Lemma 7 . Indeed, suppose for example that $t_{j}=t_{j+1}$. Then the quadrature formula (1.1) has total mass $2(1-\alpha) / p$ at $t_{j}$. Then (3.1) implies that $2(1-\alpha) / p \leq 2 /(p+1)$, which cannot hold if $\alpha<1 /(p+1)$. Similarly, if we suppose that $0=t_{1}$ or $t_{p}=1$, then we find a contradiction with $(3.2)$.

Remark. From the proof of Theorem 2 we see that for $\alpha<1 /(p+1)$ the nodes $t_{1}, \ldots, t_{p}$ are mutually distinct and distinct from the end points. This implies that the zeros of the Faber polynomial $F_{n}$ generated by the $m$-star are also mutually distinct (except for the zero at the origin).

\section{REFERENCES}

1. J. Bartolomeo and M. He, On Faber polynomials generated by an m-star, Math. Comp. 62 (1994), 277-287. MR 94c:30006

2. A. Ghizzetti and A. Ossicini, Quadrature formulae, Birkhäuser, Basel, 1970. MR 42:4012

3. S. Karlin and W. J. Studden, Tchebycheff systems: with applications in analysis and statistics, Interscience, New York, 1966. MR 34:4757

4. A. B. J. Kuijlaars, Chebyshev type quadrature and zeros of Faber polynomials, J. Comput. Appl. Math. (to appear).

5. F. Peherstorfer, Weight functions which admit Tchebycheff quadrature, Bull. Austral. Math. Soc. 26 (1982), 29-38. MR 84k:65025

6. I. J. Schoenberg and G. Szegö, An extremum problem for polynomials, Compositio Math. 14 (1960), 260-268. MR 24:A380

7. J. L. Ullman, A class of weight functions that admit Tchebycheff quadrature, Michigan Math. J. 13 (1966), 417-423. MR 34:5290

Faculteit Wiskunde en Informatica, Universiteit van Amsterdam, Plantage MuiderGracht 24, 1018 TV Amsterdam, The Netherlands

E-mail address: arno@fwi.uva.nl 